\title{
Ivermectin induces apoptosis of esophageal squamous cell carcinoma via mitochondrial pathway
}

Nana $\mathrm{Xu}^{1 \dagger}$, Mengmeng $\mathrm{Lu}^{1+}$, Jiaxin Wang ${ }^{1 \dagger}$, Yujia $\mathrm{Li}^{1}$, Xiaotian Yang ${ }^{1}$, Xiajie Wei ${ }^{1}$, Jiaoyang $\mathrm{Si}^{1}$, Jingru Han ${ }^{1}$, Xiaojuan Yao', Juanmei Zhang ${ }^{1}$, Junqi Liu ${ }^{2}$, Yanming Li ${ }^{3 *}$, Hushan Yang ${ }^{4^{*}}$ and Dengke Bao ${ }^{{ }^{*}}$

\begin{abstract}
Background: Esophageal squamous cell carcinoma (ESCC) is the most predominant primary malignant tumor among worldwide, especially in China. To date, the successful treatment remains a mainly clinical challenge, it is imperative to develop successful therapeutic agents.
\end{abstract}

Methods: The anti-proliferative effect of ivermectin on ESCC is investigated in cell model and in nude mice model. Cell apoptosis was assessed using flow cytometry, TUNEL assay and western blotting. Mitochondrial dysfunction was determined by reactive oxygen species accumulation, mitochondrial membrane potential and ATP levels.

Results: Our results determined that ivermectin significantly inhibited the proliferation of ESCC cells in vitro and in vivo. Furthermore, we found that ivermectin markedly mediated mitochondrial dysfunction and induced apoptosis of ESCC cells, which indicated the anti-proliferative effect of ivermectin on ESCC cells was implicated in mitochondrial apoptotic pathway. Mechanistically, ivermectin significantly triggered ROS accumulation and inhibited the activation of NF-KB signaling pathway and increased the ratio of Bax/BCl-2.

Conclusions: These finding indicated that ivermectin has significant anti-tumour potential for ESSC and may be a potential therapeutic candidate against ESCC.

Keywords: ESCC, Ivermectin, Apoptosis, Mitochondrial, NF-KB

\section{Background}

Esophageal squamous cell carcinoma (ESCC) is one of the most common and fatal malignancies in China $[1,2]$, which is considered as an aggressive cancer due

\footnotetext{
*Correspondence: yanminglee@163.com; hushan.yang@jefferson.edu; bdkmydy12004@126.com

†Nana Xu, Mengmeng Lu and Jiaxin Wang contributed equally to this work.

${ }^{1}$ Laboratory of Cancer Biomarkers and Liquid Biopsy, School of Pharmacy, Huaihe Hospital, Henan University, Kaifeng 475004, Henan, China

${ }^{3}$ Department of Cardiology, Huaihe Hospital, Henan University, Kaifeng 475000, Henan, China

${ }^{4}$ Department of Medical Oncology, Sidney Kimmel Cancer Center, Thomas Jefferson University, Philadelphia, PA 19107, USA

Full list of author information is available at the end of the article
}

to its poor prognosis and high mortality rate with a 5 -year survival rate of only about $20 \%$ [3-5]. Since most patients diagnosed with ESCC were found to have locally advanced or metastatic disease and thus are not candidates for radical surgical resection, these patients are treated with radiotherapy and chemotherapy $[6,7]$. However, few candidate drugs that have long-term benefits for ESCC therapy and the resistance of ESCC cells to chemotherapy, which suggests that it is urgent to identify novel therapeutic alternatives or agents to improve systemic therapy for ESCC patients.

Ivermectin is a polycyclic lactone pesticide produced by streptomyces avermitilis and is found to be a broadspectrum effect against parasites [8, 9]. Moreover, some 
studies had revealed the anti-proliferative potential of ivermectin in colon cancer, ovarian cancer, melanoma and leukemia by inducing cell apoptosis and cell cycle arrest, activating necrosis pathways and suppressing tumor initiation and malignant transformation [10-12]. Previous study demonstrated that the growth of breast cancer cells can be inhibited by ivermectin by inducing autophagy [13]. Ivermectin can also inhibit the cells growth of human ovarian cancer through blocking the oncogenic kinase PAK1 [14]. However, the effect and molecular mechanism of ivermectin on ESCC growth has not been clearly determined.

Inhibiting proliferation and increasing the sensitivity of cancer cells to undergo apoptosis are considered important properties in developing novel chemotherapeutic agents [15]. Mitochondria are important mediators of tumorigenesis and targeting mitochondrial-associated apoptotic pathways is a potential therapeutic strategy for cancer [16]. In this study, we first evaluated the anti-proliferative effect of ivermectin on ESCC cells in vitro and in vivo. Our results shown that ivermectin inhibit ESCC cells growth and induce ESCC cells apoptosis through mitochondria-dependent ROS production. Further, we demonstrate that ivermectin induced mitochondrial dysfunction and ROS accumulation of ESCC cells, which subsequently inhibited the activation of NF- $\mathrm{KB}$ signaling pathway and induced ESCC cells apoptosis. These findings demonstrated that ivermectin may be a potential candidate drug for ESCC therapy.

\section{Methods}

\section{Cell culture and treatment}

Esophageal squamous carcinoma cell lines and Normal esophageal epithelial cells, KYSE-70, KYSE-30 and NE-3 were purchased from Nanjing Kebai Biotechnology Co., ltd. The cells were cultured in RPMI-1640 (10040-CVR, Corning) medium containing $10 \%$ fetal bovine serum (04-001-1Acs, $\mathrm{BI}$ ) in a $37^{\circ} \mathrm{C} 5 \% \mathrm{CO}_{2}$ incubator, and cells were grown to $65 \%-75 \%$ confluence and treated under various conditions as indicated.

\section{Cell viability, proliferation and lactate dehydrogenase (LDH) leakage assays}

ESCC cells and NE-3 were seeded in 96-well plates $\left(5 \times 10^{5}\right.$ cells/well or $1 \times 10^{5}$ cells/well $)$ for $24 \mathrm{~h}$, and treated with ivermectin (S1351, Selleck Chemicals) at serially diluted concentrations of $2.5,5,10,15,20 \mu \mathrm{M}$ on NE-3 and KYSE-70, 2, 4, 6, 8, $10 \mu \mathrm{M}$ on KYSE-30 for 48 $\mathrm{h}$. We found that the half maximal-inhibitory concentration $\left(\mathrm{IC}_{50}\right)$ of ivermectin for KYSE-70 and KYSE-30 cells was close to $10 \mu \mathrm{M}$ and $6 \mu \mathrm{M}$, respectively. Thus, $10 \mu \mathrm{M}$ and $6 \mu \mathrm{M}$ ivermectin were used for further experiments to evaluate the anti-proliferative effect on ESCC cells.
MTT assay was performed to assess the viability of ESCC as described previously [17]. ESCC cells were seeded at a density of 2000 cells/well or 500 cells/well in 6-well plates, incubated for $24 \mathrm{~h}$, and then exposed to ivermectin for 10-14 days. Next, the medium was removed and cells were fixed with $4 \%$ paraformaldehyde for $15 \mathrm{~min}$, washed three times with PBS and incubated with $0.5 \%$ crystal violet solution (C8470, Soiarbio) for another 5 min. The colonies were counted and assays from three independent experiments. EdU (C10310, Ribobio) incorporation assay were used to evaluate the proliferation of cells according to the manufacturer's instructions. Then results were analyzed with a fluorescence microscope (Olympus Corporation U-LH100HG). LDH leakage was measured using a colorimetric LDH assay kit (C0017, Beyotime) following the manufacturer's recommended protocol. For relative quantification, the value of absorbance in each group was normalized to the control group. Cell cycle of ESCC cells after treatment with ivermectin is analyzed using Cell cycle kit (C6031, US Everbright) following manufacturer's instructions and determined by flow cytometry using CytoFLEX (Beckman Coulter).

\section{Cell apoptosis assay}

Cell apoptosis was assessed using the Annexin-V-FITC apoptosis detection kit (C6031, US Everbright). ESCC cells were seeded in 6-well plates for $24 \mathrm{~h}$, and treated with ivermectin at $5,10,15 \mu \mathrm{M}$ on KYSE-70 and 4,6 , $8 \mu \mathrm{M}$ on KYSE-30 for $48 \mathrm{~h}$, the apoptosis was assessed following the manufacturer's protocol and analyzed by a flow cytometry (FACSVerse, BD), and the data were analyzed with Flowjo VX10 Software (VX10, USA). Cell apoptosis was also detected by using the TUNEL assay kit (T6013, UE) and Hoechst 33342 (H4047, UE) staining following the manufacturer's protocol. The cellular nuclei were stained with the TUNEL reaction overnight at $4{ }^{\circ} \mathrm{C}$ and fixed with $4 \%$ paraformaldehyde for $5 \mathrm{~min}$. After washing three times using PBS, the cells were incubated with Hoechst 33342 for $10 \mathrm{~min}$. The TUNEL-positive cells (green) and Hoechst 33342 (blue) staining patterns were detected by fluorescence microscope (Olympus Corporation U-LH100HG).

\section{Detection of reactive oxygen species, mitochondrial membrane potential (MMP) and ATP production}

The cells were planted in 6-well plates and cultured overnight, exposed to ivermectin at $37^{\circ} \mathrm{C}$ for $48 \mathrm{~h}$. Cellular reactive oxygen species (ROS) levels were detected by the fluorescent probe DCFH-DA following the manufacturer's protocols (S0033S, Beyotime) [18]. Briefly, ESCC cells suspension were incubated with $10 \mu \mathrm{M}$ DCFH-DA for $20 \mathrm{~min}$ at $37^{\circ} \mathrm{C}$ and analyzed by a flow cytometry (FACSVerse, BD), and the data were analyzed with Flowjo VX10 
Software (VX10, USA). The alteration of cellular MMP was evaluated using JC-1 kit (C2006, Beyotime). Cells were adjusted to a density of $5 \times 105 / \mathrm{ml}$ and stained with $2 \mu \mathrm{g} / \mathrm{ml} \mathrm{JC}-1$ dye for $20 \mathrm{~min}$ at $37^{\circ} \mathrm{C}$, the results were observed by a fluorescence microscope (Olympus Corporation U-LH100HG). The total ATP levels were determined using the Cell Titer-Glo Luminescent assay (FF2000, Promega) according to the manufacturer's instructions.

\section{Subcutaneous xenograft models}

5-week-old female Balb/c nude mice (Beijing Charles River Laboratories, Beijing, China) were randomly divided into indicated groups $(\mathrm{n}=5) .5 \times 10^{6}$ ESCC cells in $20 \mu \mathrm{L}$ serum free RPMI-1640 was injected into the right flanks to establish the subcutaneous xenograft nude mice model. Tumor size was measured every two days, and the tumor volume was calculated according to (Width $2 \times$ Length) $/ 2$. When the tumor volumes reached $100 \mathrm{~mm}^{3}$, mice were randomized into two groups receiving $0.1 \mathrm{~mL}$ of saline or $25 \mathrm{mg} / \mathrm{kg}$ ivermectin per nude mice, respectively. The nude mice were monitored twice a week for palpable tumor formation. Then, all mice were euthanized by intravenous with $100 \mathrm{mg} / \mathrm{kg}$ sodium pentobarbital (P3761, Sigma) and the tumors were harvested and weighted. All experiments in this study were performed in accordance with relevant guidelines and regulations and were approved by Ethics Committee of Henan University. Animal experiments were carried out in compliance with the ARRIVE guidelines and was approved by the Institutional Animal Care and Use Committee of Henan University (HUSOM-2019-031).

\section{qPCR, Western blot, IHC}

Total RNA extraction, complementary DNA synthesis, and qPCR were performed as previously described [19]. Primer sequences of qPCR were provided in Supplementary Table 2. Preparation of whole-cell protein lysates and western blotting analysis were performed as we described before $[17,20]$. Primary antibodies used in this study included anti-P65 (8242T; D14E12; Cell Signal-

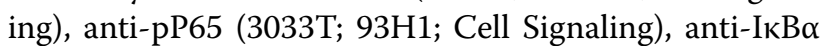
(4814T; L35A5; Cell Signaling), anti-pIkB $\alpha$ (2859T; 14D4; Cell Signaling), anti-PARP-1 ( sc-56197; 194C1439; Santa Cruz Biotechnology), anti-cleaved PARP-1 (sc-56196; 5A5; Santa Cruz Biotechnology), anti-Bcl-2 (12789-1-AP; Polyclonal Antibody), anti-Bax(50599-2-lg; Polyclonal Antibody), anti-Cleaved-caspase-3 (CY5031; Abways), and anti-Cleaved-caspase-9 (CY5682; Abways). The IHC staining (D601037, Sangon Biotech) was detected by using immunohistochemistry kit according to the manufacturer's instructions. The cleaved caspase 3 (CY5031; Abways), ROS1 (ab189925; EPMGHR2; Abcam), p-p65
(3033T; Cell Signaling) and Ki-67 (CY5542; Abways)-positive staining was detected using a microscope (Nikon, E100) and quantification using the ImageJ software (NIH, Bethesda, MD, USA). Details of reagents used in this study were provided in Supplementary Table 1.

\section{Statistical treatment}

The results were expressed as the mean $\pm \mathrm{SD}$. Mean values were calculated from data obtain from experiments performed in triplicate. The differences between the experimental and control groups were compared using one-way ANOVA followed by Dunnett's multiple comparisons test. Statistical software SPSS20.0 was used in data processing and for analyzing the significance between groups with the $\mathrm{t}$-test. $p<0.05$ was considered statistically significant.

\section{Results}

\section{Ivermectin inhibits proliferation of ESCC cells in vitro}

To investigate the anti-proliferative effect of ivermectin on ESCC cells, the MTT assay was conducted to assess the growth of ESCC cells after incubated with ivermectin for 48h. As shown in Fig. 1A, ivermectin markedly decreased the cell viability of ESCC cells with a dosedependent manner. Moreover, the half maximal-inhibitory concentration $\left(\mathrm{IC}_{50}\right)$ of ivermectin for KYSE-70 and KYSE-30 cells was close to $10 \mu \mathrm{M}$ and $6 \mu \mathrm{M}$, respectively. We also found that the typical morphological characteristic of ESCC cells was changed after ivermectin treatment for 48h. The morphology of ESCC cells with ivermectin treatment became irregular, wrinkled or even broken and smaller and roundness than the control group (Fig. 1B). Lower concentrations of ivermectin $(2.5-15 \mu \mathrm{M})$ had no cytotoxic effect in NE-3 cells, while $20 \mu \mathrm{M}$ ivermectin slightly inhibited viability of NE-3 cells viability (Fig. 1A). We next performed another cytotoxicity evaluation in ESCC cells after ivermectin treatment using the LDH cytotoxicity assay kit after ivermectin treatment. As shown in Fig. 1C, treatment with ivermectin significantly increased LDH release in the medium, which was consistent with MTT assay. The anti-proliferative effect of ivermectin on ESCC cells was determined by colony formation and EdU incorporation assays. As shown in Fig. 1D, the proliferative potential and colony formation ability of ESCC cells were remarkably suppressed after ivermectin treatment. EdU incorporation was remarkably reduced in ivermectin-treated ESCC cells compared to controls (Fig. 1E). Cell cycle analysis performed using flow cytometry indicated that ivermectin significantly decrease the population of cells in S and G2/M phase and increase the population of G1 with a concentrationdependent manner (Fig. 1F). Collectively, these results 


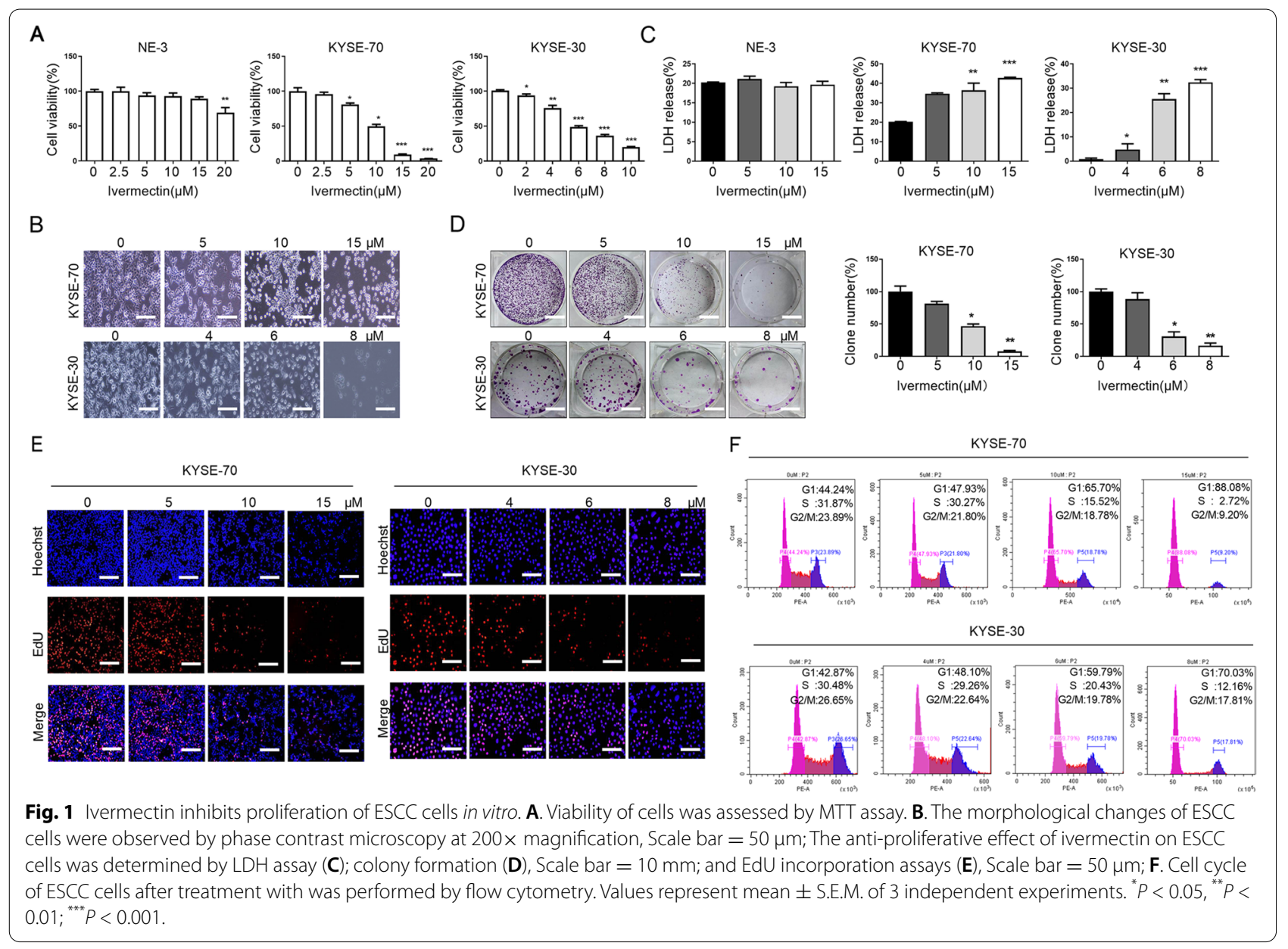

demonstrate that ivermectin inhibits the proliferation of ESCC cells in vitro.

\section{Ivermectin promotes apoptosis of ESCC cells}

Apoptosis is a major form of cell death for cancer cells induced by chemotherapeutic agents and implicated in the cytotoxic mechanisms of various classes of chemotherapy [21]. Therefore, we first simultaneously evaluated the expression level of Bax and $\mathrm{Bcl}-2$, two apoptosis related proteins, and analyzed the ratio of $\mathrm{Bax} / \mathrm{Bcl}-2$, simultaneously. As shown in Fig. 2A and $\mathrm{B}$, the expression level of pro-apoptotic factor Bax was significantly increased after ivermectin treatment, while the expression of anti-apoptotic factor Bcl-2 was markedly decreased when compared with control group. Thus, the ratio of Bax/Bcl-2 was significantly increased in ivermectin-treated ESCC cells. The activation of the caspase cascade is well known intrinsic cell apoptotic pathway via mitochondria. The expression levels of cleaved-caspase- 9 , cleaved-caspase- 3 and caspase downstream effectors (PARP) were estimated by western blot analysis. As shown in Fig. 2C, after ivermectin treatment, the levels of cleaved-caspase 9, cleaved-caspase 3 and cleaved-PARP protein were remarkably increased. We further detected whether ivermectin induced apoptosis in the ESCC cells by Hoechst 33342 staining assays and flow cytometry analysis using Annexin V-FITC/PI kit. Hoechst 33342 staining assays showed that the nuclear morphology of ESCC cells exposure to ivermectin were condensed, fragmented and crescent shaped, while the control cells had a round, bright and regular morphology (Fig. 2D). Using TUNEL and Annexin-V FITC/PI assays by flow cytometry, we showed that the percentage of apoptotic ESCC cells was significantly increased in the ivermectin group compared to control (Fig. 2E and F). Taken together, these data suggested that the inhibitory effect of ivermectin on ESCC cells was associated with cell apoptosis.

\section{Ivermectin mediates mitochondrial dysfunction of ESCC cells}

Intracellular ROS, which are predominantly derived from the mitochondria, are known to induce oxidative damage and ultimately trigger a series of mitochondrial associated events, including apoptosis. Consistent with 


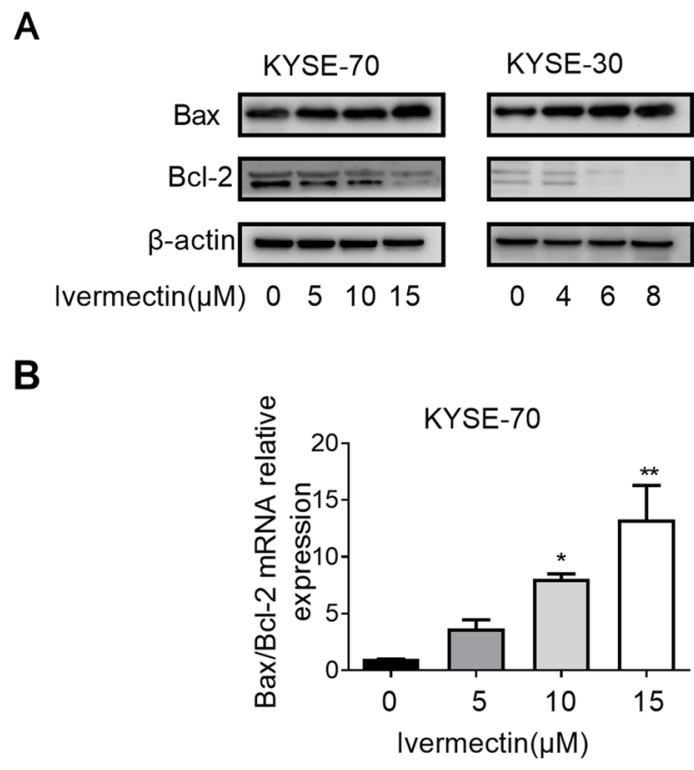

C

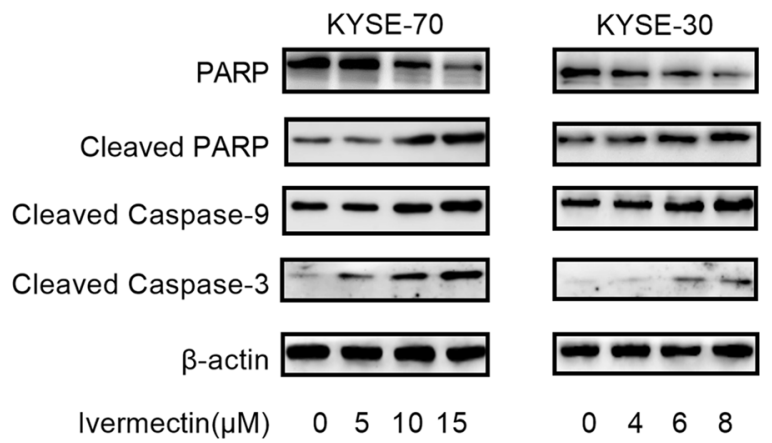

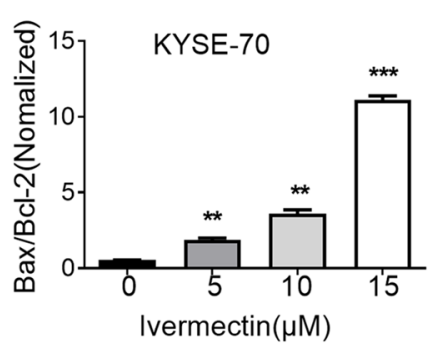
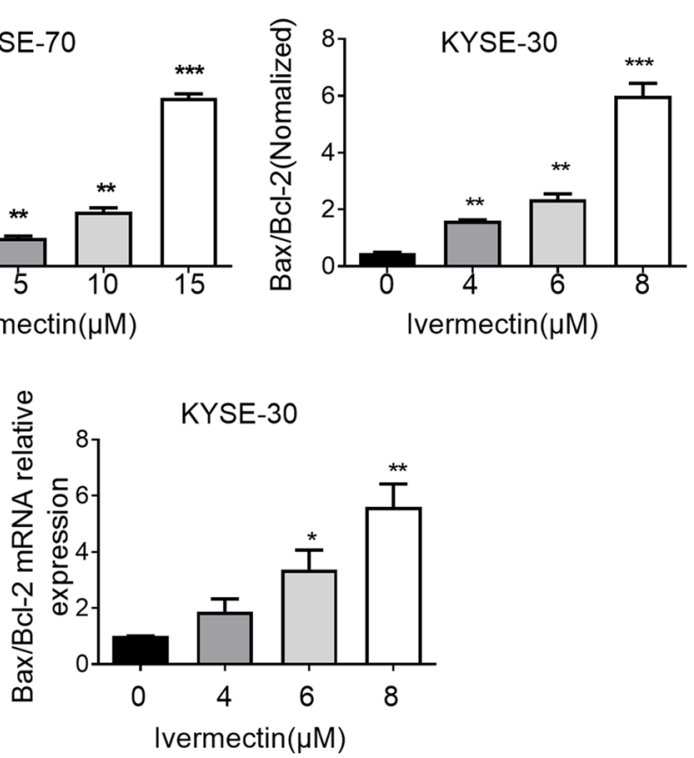

D

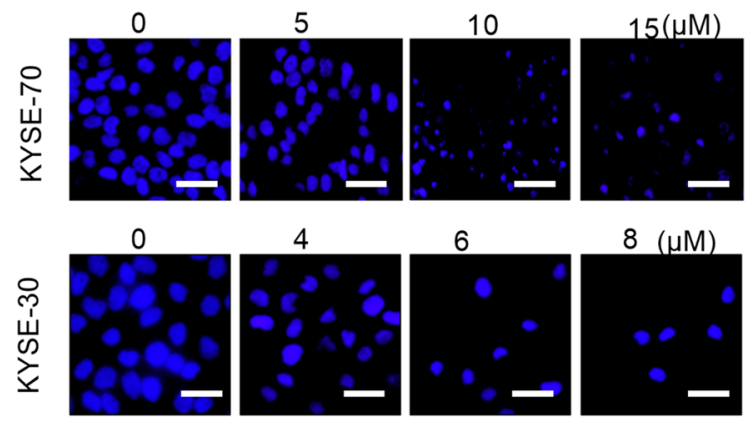

E

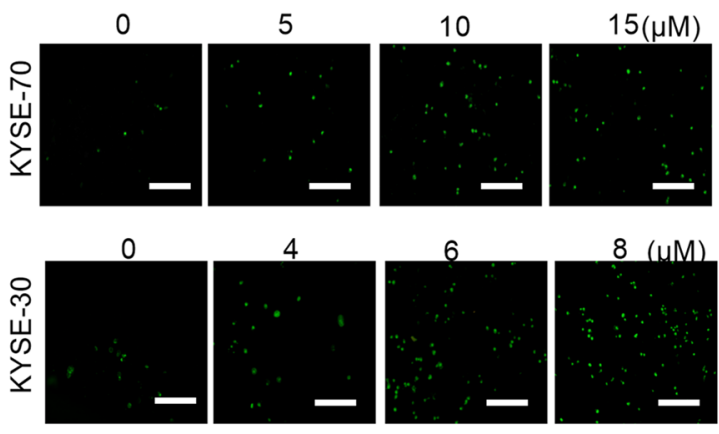

$\mathbf{F}$

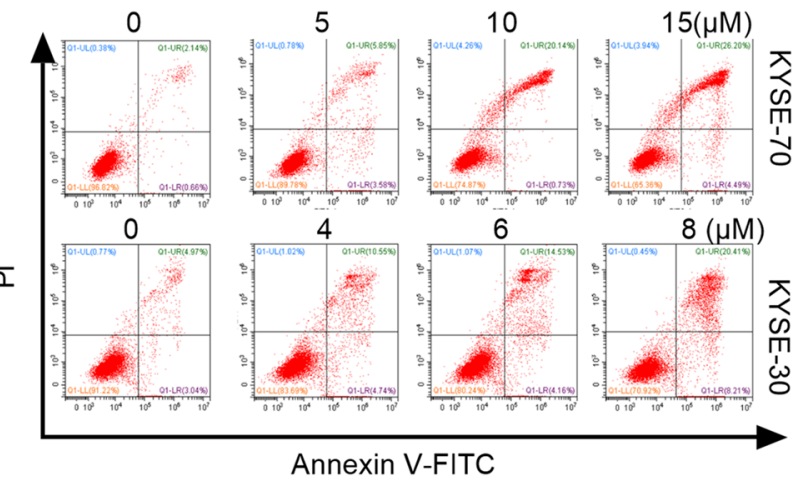

Fig. 2 Ivermectin promotes apoptosis of ESCC cells. Western blotting (A) and qRT-PCR (B) analysis for the expression levels of Bax and Bcl-2 after ivermectin treatment; $\mathbf{C}$. The expression of caspase cascade (cleaved-caspase 9, cleaved-caspase 3, PARP, cleaved PARP) were analyzed by western blotting; D. Cells were observed by fluorescence microscopy after staining with Hoechst 33342, Scale bar = $100 \mu$ m; E. Ivermectin-induced ESCC cells apoptosis was determined by TUNEL assay $(200 \times)$, Scale bar $=50 \mu \mathrm{m} ; \mathbf{F}$. Ivermectin-induced ESCC cells apoptosis was determined by PI/ Annexin-V assay using flow cytometry. ${ }^{*} P<0.05,{ }^{* *} P<0.01 ;{ }^{* * *} P<0.001$. 


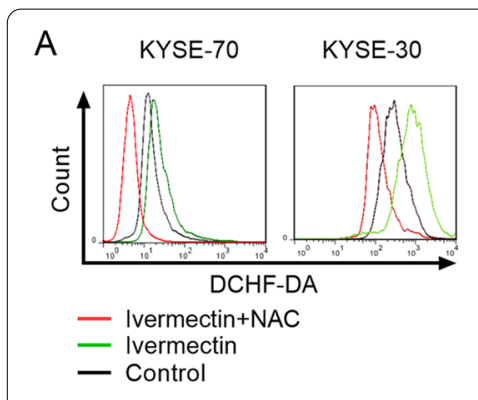

C

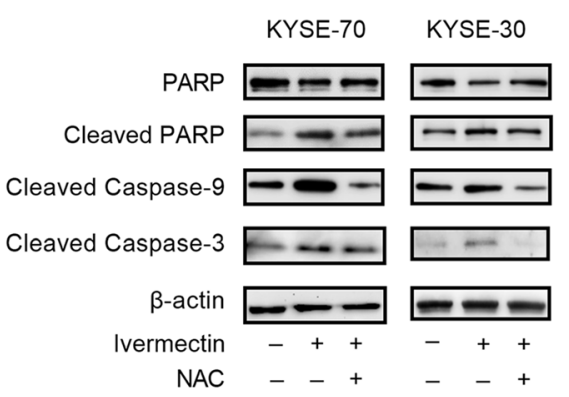

$\mathrm{E}$

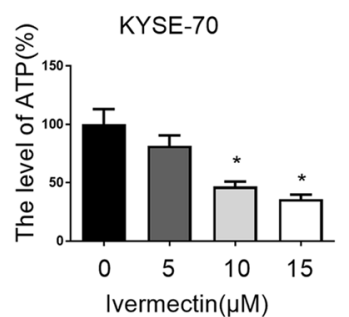

B

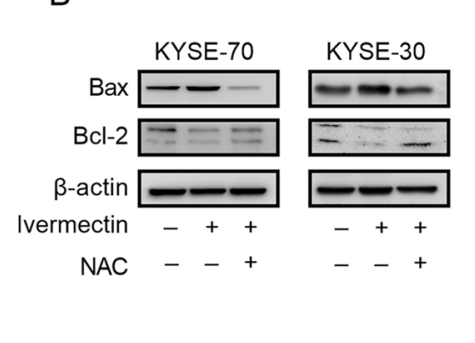

D
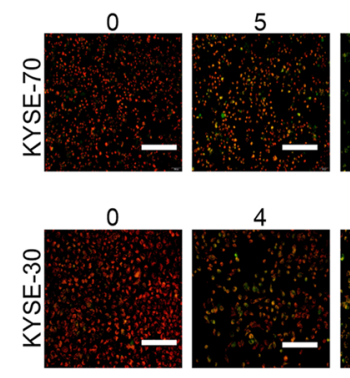

$\mathrm{F}$

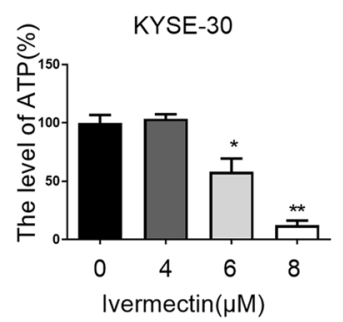

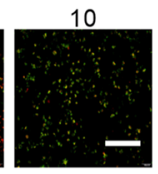
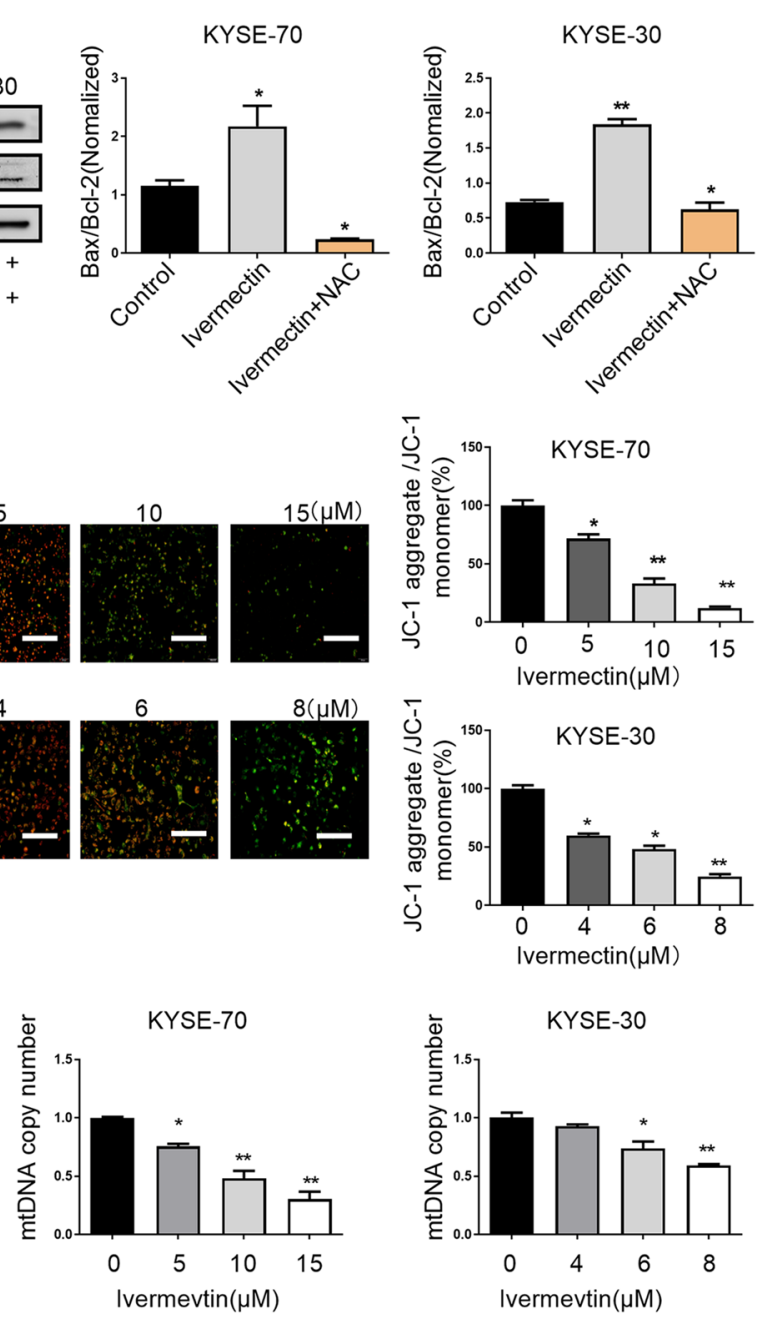

Fig. 3 Ivermectin mediates mitochondrial dysfunction of ESCC cells. A. Intracellular ROS levels were investigated by DCFH-DA fluorescence in ESCC cells treatment with indicated; $\mathbf{B}$. The expression levels of Bax and Bcl-2 were determined by western blot analysis; $\mathbf{C}$. The expression of caspase cascade (cleaved-caspase 9, cleaved-caspase 3, PARP, cleaved PARP) were analyzed by western blotting; $\mathbf{D}$. Mitochondrial membrane potential was observed by fluorescence microscope at $200 \times$ magnifications, Scale bar $=50 \mu \mathrm{m}$; E. ATP production of ESCC cells after treated with ivermectin was detected; $\mathbf{F}$. The mitochondrial DNA copy number in ESCC cells treatment with invermectin was evaluated using qRT-PCR. ${ }^{*} P<0.05,{ }^{* *} P<0.01$.

previous research, we found that ivermectin induced intracellular ROS accumulation in ESCC cells [12]. To determine whether the ROS accumulation was functionally important for ivermectin-induced apoptosis, we added the free radical scavenger $\mathrm{N}$-acetylL-cysteine (NAC) to ivermectin-treated cells. NAC markedly eliminated intracellular ROS induced by ivermectin (Fig. 3A) and abrogated ivermectin-induced the increase of $\mathrm{Bax} / \mathrm{Bcl}-2$ ratio (Fig. $3 \mathrm{~B}$ ). Consistent with this data, the expression of cleaved caspase 9, cleaved caspase 3 and cleaved PARP proteins in ESCC cells were significantly decreased after exposure to ivermectin and NAC, when compared with ivermectin alone (Fig. 3C).
It had been demonstrated that mitochondriadependent apoptotic pathway play a vital role in drug-induced apoptosis [22]. To determine whether ivermectin induced ESCC cells apoptosis though a mitochondria-dependent pathway, we examined the mitochondrial function by mitochondrial membrane potential, mitochondrial DNA contents change and ATP production. As shown in Fig. 3D, we found that more ESCC cells with green or orange fluorescence, which indicated that mitochondrial membrane potential was decreased after ivermectin treatment. Ivermectin significantly decreased ATP production in ESCC cells with a time-dependent manner (Fig. 3E). To further verify the hypothesis, the mitochondrial 
DNA contents change in ESCC cells were detected after ivermectin treatment. Simultaneity, our results indicated that mitochondrial DNA contents were significantly reduced in ESCC cells treatment with ivermectin (Fig. 3F). These results indicated that ivermectin-induced ESCC cells apoptosis was associated with mitochondrial dysfunction.

\section{Ivermectin induces apoptosis of ESCC cells through NF-KB pathway}

The NF- $\kappa$ B signaling pathway is involved in ESCC carcinogenesis and progression and is hyperactivated in ESCC cells and promotes cell survival. To evaluate whether NF- $\mathrm{kB}$ signaling pathway was implicated in ivermectin-mediated anti-tumor properties of ESCC, the expression of related signaling molecules in NF- $\mathrm{kB}$ pathway were measured. We observed that ivermectin significantly decreased the phosphorylation and nuclear translocation of NF-kB p65 protein in a concentration-dependent manner (Fig. 4A and B). Subsequently, we also found that the phosphorylation of $\mathrm{I} \kappa \mathrm{B} \alpha$, an upstream regulatory molecule of NF-kB pathway, was inhibited in ESCC cells after ivermectin treatment (Fig. 4A and B). Consistent with previous study, eliminating intracellular ROS by NAC markedly abrogated ivermectin-induced inactivation of $N F-\kappa B$ pathway, observed through the increased phosphorylation of $\mathrm{I} \kappa \mathrm{B} \alpha$ and NF-kB p65 protein (Fig. 4C and D). Taken together, these results indicated that ivermectin induces apoptosis of ESCC cells through NF-kB pathway.

\section{Ivermectin suppressed xenograft growth of ESCC cells in vivo}

To evaluate the anti-proliferative effects of ivermectin on ESCC growth in vivo, xenograft nude mice model was established by subcutaneously injecting ESCC cells. We observed that the growth potential of ESCC cells-derived subcutaneous tumor was remarkably attenuated by treatment with ivermectin. The size of xenografts tumor treated with ivermectin was significantly smaller than control saline groups (Fig. 5A) and the proliferation rate in ivermectin treatment group was slower (Fig. 5B). Tumor weight was also markedly reduced in ivermectin-treated mice when compared to control group (Fig. 5C). Furthermore, the percentage of Ki67-positive cells in xenografts tumor by injection of ivermectin was also notably attenuated (Fig. 5D and E). The p-p65 expression in nuclear was

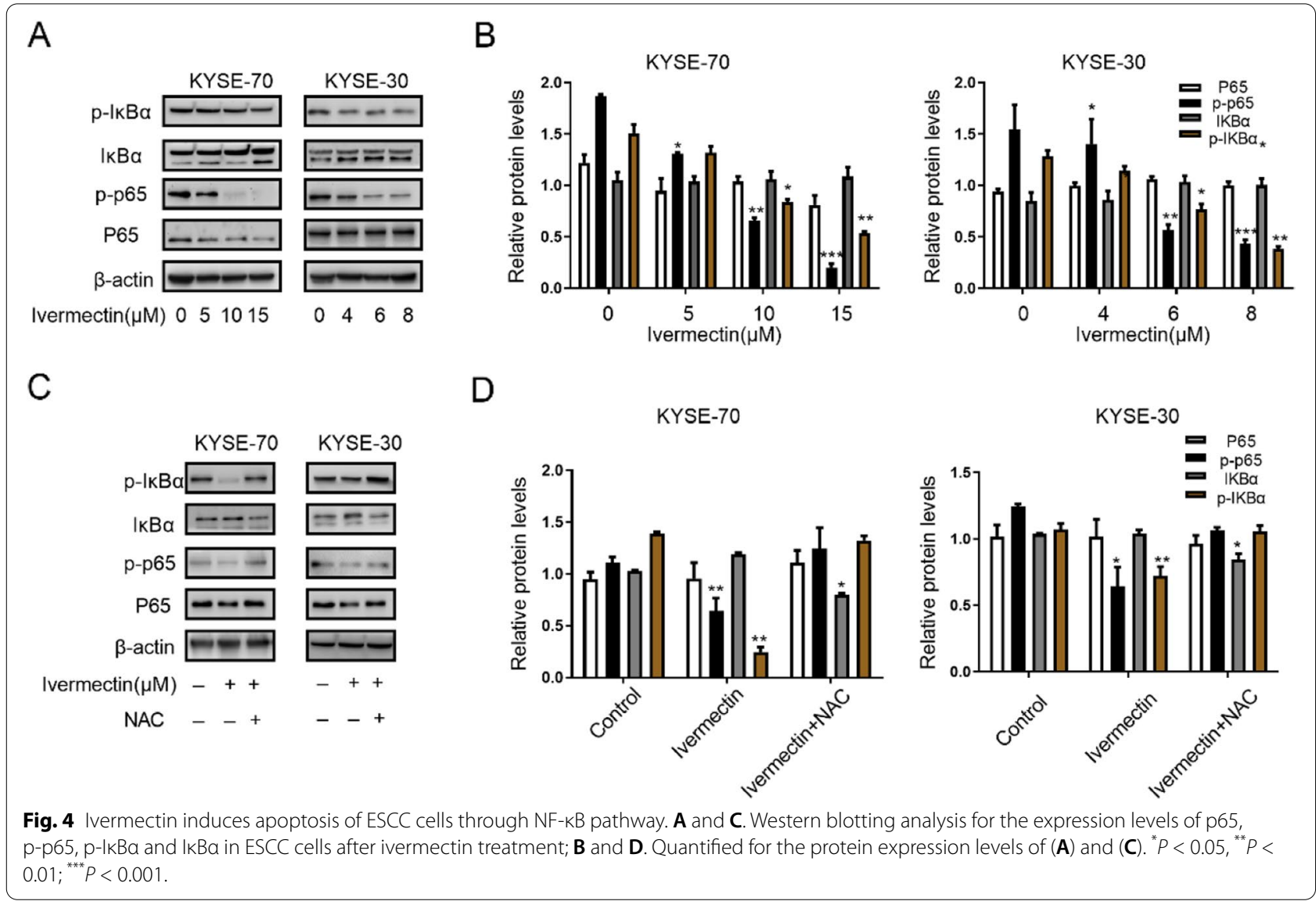




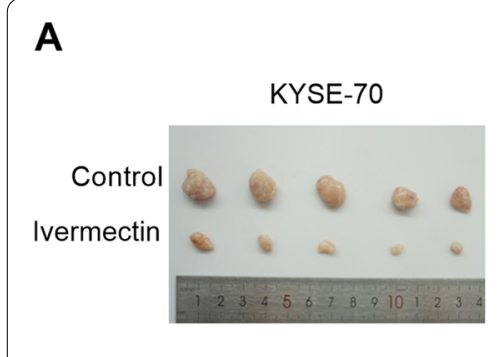

C

B
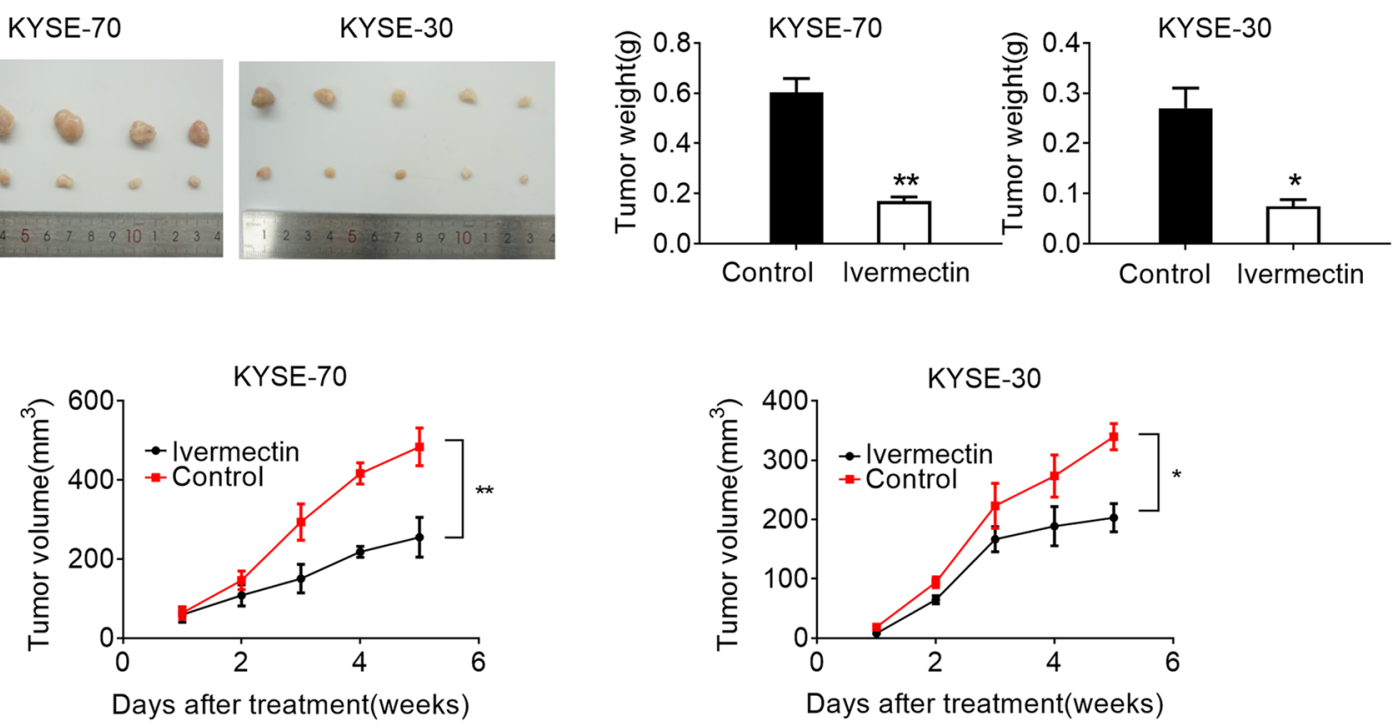

D
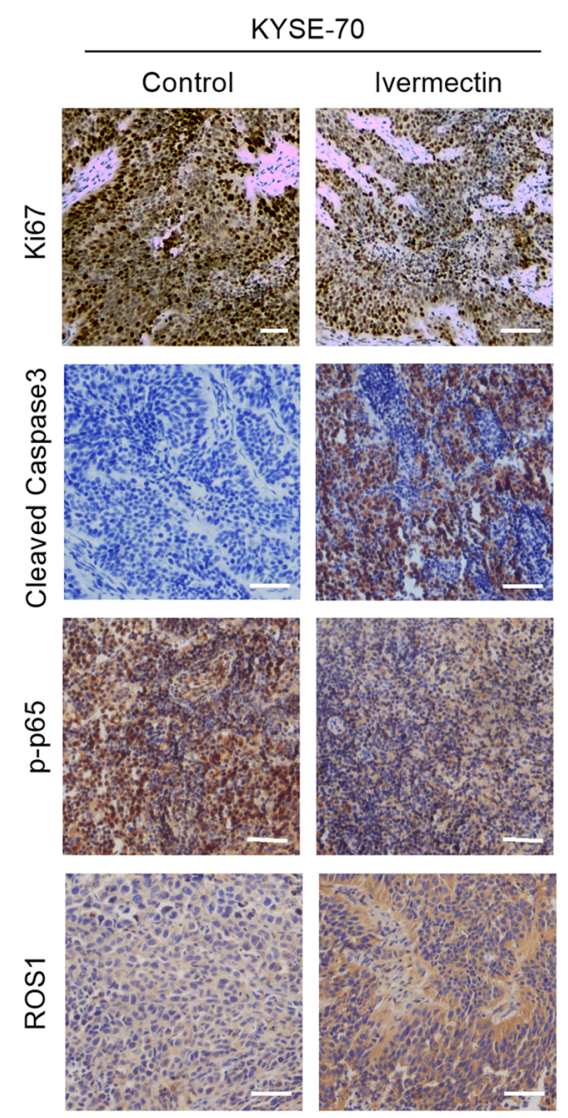
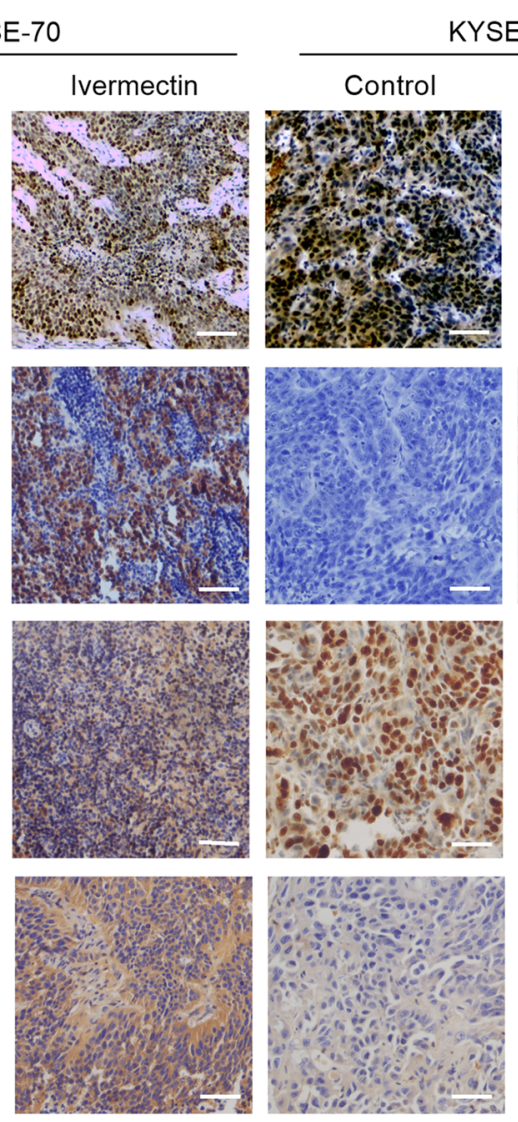

KYSE-30

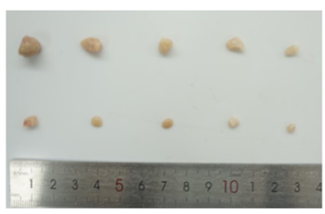

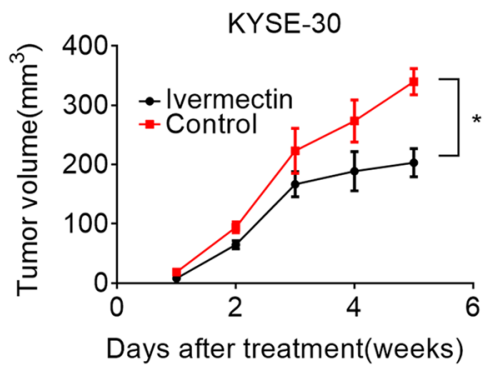

E
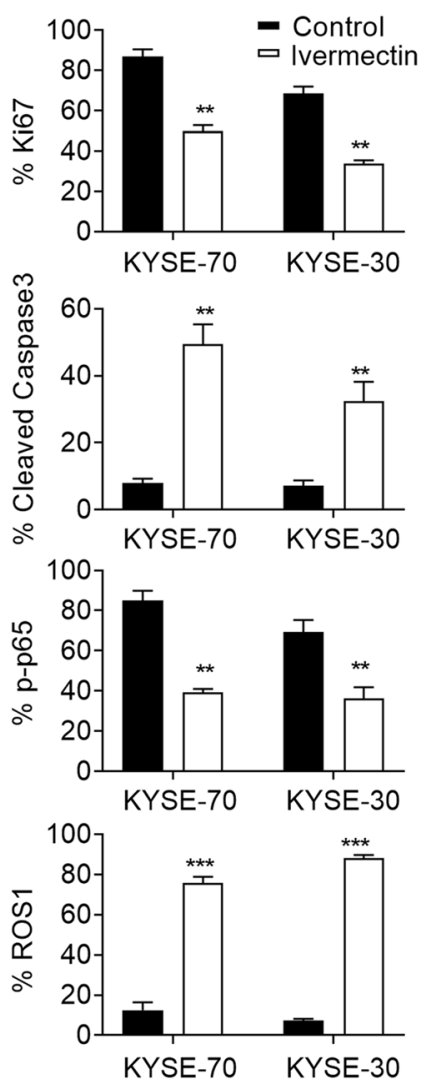

Fig. 5 Ivermectin suppressed xenograft growth of ESCC cells in vivo. A, B and C. Tumor growth curves and weight of subcutaneous xenograft tumor model developed from ESCC cells treatment with ivermectin as indicated $(n=5) ; \mathbf{D}$ and $\mathbf{E}$. Representative immunohistochemistry images of Ki67, ROS1, p-p65 and cleaved-caspase 3 in xenograft tumor developed from KYSE-70 and KYSE-30 cells treatment with ivermectin as indicated. Scale bar: $300 \mu \mathrm{m} .{ }^{*} P<0.05,{ }^{* *} P<0.01 ;^{* * *} P<0.001$. 
significantly decreased in xenografts tumor cells by injection of ivermectin (Fig. 5D and E). However, the expression of ROS1 and cleaved caspase 3 were remarkable increased in ivermectin treatment group (Fig. 5D and E). Taken together, these data suggest that ivermectin inhibited the growth of ESCC cells both in vivo.

\section{Discussion}

In this study, the anti-proliferative potential of ivermectin on ESCC cells was evaluated. Our results demonstrated that ivermectin could inhibit the proliferation of ESCC cells in vivo and in vitro. Furthermore, we found that ivermectin induced apoptosis of ESCC cells, which was implicated with mitochondrial pathway. In addition, the apoptosis induced by ivermectin triggered by mitochondrial dysfunction-derived ROS through NF- $\mathrm{KB}$ signaling pathway (Fig. 6).

Recent studies demonstrated that antiparasitic drugs can inhibit the growth of multiple cancers, such as melanoma, ovarian cancer, breast cancer, glioblastoma [11, 13, 23, 24]. Here, we found that treatment with ivermectin conspicuously suppressed the proliferation of ESCC cells by MTT, colony formation and EdU incorporation assay and were demonstrated in vivo of xenografts tumor. All control xenografts displayed stronger Ki67 staining than that of ivermectin-treated mice. Taken together, these data suggest that ivermectin inhibits the growth of esophageal cancer both in vitro and in vivo. It had been reported that ivermectin inhibited breast and ovarian cancer cells proliferation by promoting PAK1 ubiquitination degradation and cytostatic autophagy by suppressing Akt/mTOR signaling pathway [13, 14]. The anti-proliferative effect of ivermectin on glioma cells was implicated with ivermectinindcued cell cycle arrest and apoptosis [23]. Here, our results on ESCC cells indicated that the anti-proliferative effect of ivermectin on ESCC was significantly mediated via apoptosis. We found that treatment with ivermectin significantly increased nuclei fragmentation, apoptosis and G1 cycle arrest in ESCC cells.

NF- $\mathrm{KB}$ signaling pathway has obvious functions of inhibiting apoptosis, promoting cell proliferation and immune activation, and is also closely related to the differentiation, invasion and migration of several tumor cells [25]. Previous study had demonstrated that NF- $K B$ activation promoted transcription of Bcl-2, VEGF, MMP and inhibited Bax expression, which reduced apoptosis and contributed to angiogenesis and progression of ESCC [26]. Blocking of NF- $\kappa B$ signaling pathway significantly suppressed ESCC cells growth, prevented angiogenesis and metastasis of ESCC, and sensitized ESCC to chemotherapeutic drugs $[27,28]$. We also found that NF- $\mathrm{kB}$ pathway was markedly suppressed after treatment with ivermectin. Consistent with previous research,

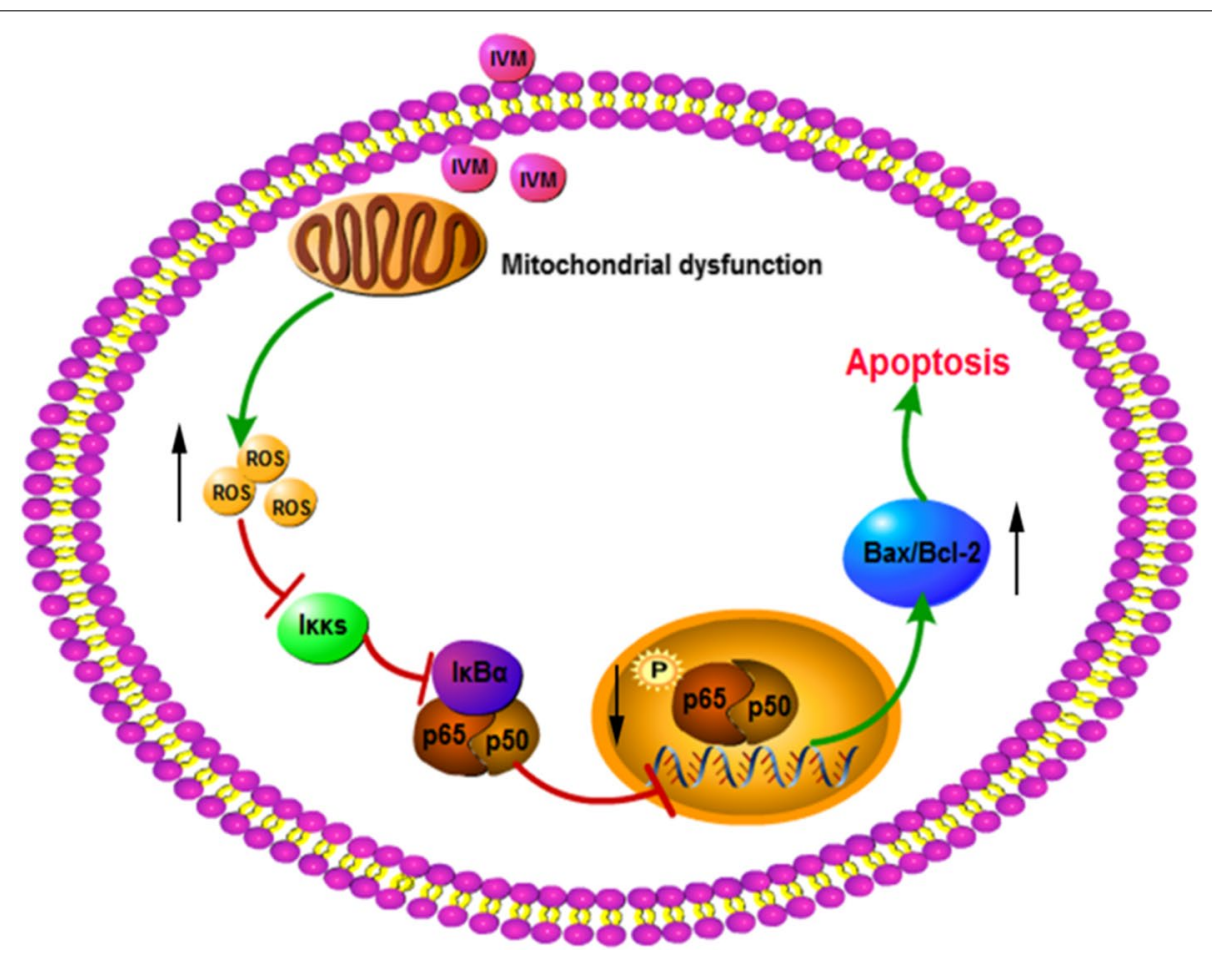

Fig. 6 Schematic depicting the anti-proliferative potential of ivermectin on ESCC cells. 
our results indicated ivermectin inhibited the growth of ESCC cells by regulating the NF- $\mathrm{kB}$ signaling pathwaymediated apoptosis, at least in part, mediated by regulation of Bax and Bcl-2 expression. The above results show that ivermectin, as an external stimulus signal, blocks further activation of the NF- $\mathrm{kB}$ signaling pathway, thereby attenuating the anti-apoptotic effect, and the downstream apoptotic signal further promotes apoptosis.

Mitochondrion is the central of cytosolic signaling transduction and plays a vital role in apoptotic pathway and is implicated in several anticancer drugs $[22,29,30]$. Recently research determined that ivermectin suppresses tumour growth and metastasis through degradation of PAK1 in ESCC [31]. However, how the mitochondria is involved in ivermectin-induced apoptosis remains unclear. Here, we determined mitochondrial dysfunction in ESCC cells treated with ivermectin, seen in the reduced mitochondrial DNA contents, decreased mitochondrial membrane potential and inhibited ATP production. Ivermectin markedly increased ROS levels of ESCC cells and scavenging the ROS by NAC surprisingly blocked the apoptosis and NF- $\mathrm{KB}$ inactivation, suggesting that ROS-mediated apoptosis was the main mechanism for ivermectin activity, while other pathways may also play a role to be explored in the future. The results indicated that mitochondrial dysfunction-derived ROS maybe trigger NF- $\mathrm{kB}$ signaling pathway and induce apoptosis by ivermectin treatment.

\section{Conclusions}

We demonstrated that ivermectin effectively inhibit the proliferation of ESCC cells by inducing mitochondrial dysfunction, suppressing NF- $\mathrm{kB}$ signaling and promoting apoptosis. Our results suggest that ivermectin may be a potential therapeutic target against ESCC.

\section{Abbreviations}

ESCC: Esophageal squamous cell carcinoma; LDH: Proliferation and lactate dehydrogenase; EdU: Ethynyl deoxyuridine; MMP: Mitochondrial membrane potentialCellular; ROS: Reactive oxygen species; IC50: The half maximal-inhibitory concentration; NAC: Free radical scavenger $\mathrm{N}$-acetyl-L-cysteine.

\section{Supplementary Information}

The online version contains supplementary material available at https://doi. org/10.1186/s12885-021-09021-x.

Additional file 1. The original blots to related Fig. 2. A. The original blots to related Fig. 2A; B. The original blots to related Fig. 2 C.

Additional file 2. The original blots to related Fig. 3. A. The original blots to related Fig. 3B; B. The original blots to related Fig. 3 C.

Additional file 3. The original blots to related Fig. 4. A. The original blots to related Fig. 4A; B. The original blots to related Fig. 4C.

Additional file 4.
Acknowledgments

Not applicable

\section{Authors' contributions}

XNN wrote the manuscript. LMM, LYJ, YXT, WJX, and LJQ performed some of the lab work and data collection. WXJ, SJY, HJR and YXJ supported the overall data analysis and provided constructive discussion. XNN, ZJM and BDK performed the animal studies. BDK, YHS and LYM conceived and designed the study. All authors read and approved the final manuscript

\section{Funding}

This work was supported by the Medical Scientific and Technological Project of Henan Province in China (NO. SB201902030); Project funded by China Postdoctoral Science Foundation (NO. 2020M682286) and Science and Technology Development Program of Kaifeng (NO. 2003008).

\section{Availability of data and materials}

The datasets used in the current study are available from the corresponding author on reasonable request.

\section{Declarations}

Ethics approval and consent to participate

All experiments in this study were performed in accordance with relevant guidelines and regulations and were approved by Ethics Committee of Henan University. Animal study was carried out in compliance with the ARRIVE guidelines and was approved by the Institutional Animal Care and Use Committee of Henan University.

\section{Consent for publication}

Not applicable.

\section{Competing interests}

The authors declare that there is no conflict of interest.

\section{Author details}

${ }^{1}$ Laboratory of Cancer Biomarkers and Liquid Biopsy, School of Pharmacy, Huaihe Hospital, Henan University, Kaifeng 475004, Henan, China. ${ }^{2}$ Department of Radiation Oncology, the First Affiliated Hospital of Zhengzhou University, Zhengzhou 450052, Henan, China. ${ }^{3}$ Department of Cardiology, Huaihe Hospital, Henan University, Kaifeng 475000, Henan, China. ${ }^{4}$ Department of Medical Oncology, Sidney Kimmel Cancer Center, Thomas Jefferson University, Philadelphia, PA 19107, USA.

Received: 11 March 2021 Accepted: 14 November 2021

Published online: 07 December 2021

\section{References}

1. Zou J, et al. A novel oral camptothecin analog, gimatecan, exhibits superior antitumor efficacy than irinotecan toward esophageal squamous cell carcinoma in vitro and in vivo. Cell Death Dis. 2018;9(6):661.

2. Zeng H, et al. Cancer survival in China, 2003-2005: a population-based study. Int J Cancer. 2015;136(8):1921-30.

3. Lin DC, et al. Genomic and molecular characterization of esophageal squamous cell carcinoma. Nat Genet. 2014;46(5):467-73.

4. Kano Y, et al. Novel drug discovery system for cancer stem cells in human squamous cell carcinoma of the esophagus. Oncol Rep. 2014;31(3):1133-8.

5. Zhang J, et al. Establishment and characterization of esophageal squamous cell carcinoma patient-derived xenograft mouse models for preclinical drug discovery. Lab Investig. 2014;94(8):917-26.

6. Cory S, Adams JM. The Bcl2 family: regulators of the cellular life-or-death switch. Nat Rev Cancer. 2002;2(9):647-56.

7. Dubecz A, et al. Temporal trends in long-term survival and cure rates in esophageal cancer: a SEER database analysis. J Thorac Oncol. 2012;7(2):443-7.

8. Jemal A, et al. Global cancer statistics. CA Cancer J Clin. 2011;61 (2):69-90. 
9. Gonzalez-Canga A, et al. A review of the pharmacological interactions of ivermectin in several animal species. Curr Drug Metab. 2009;10(4):359-68.

10. Drinyaev VA, et al. Antitumor effect of avermectins. Eur J Pharmacol. 2004;501(1-3):19-23.

11. Sharmeen $\mathrm{S}$, et al. The antiparasitic agent ivermectin induces chloridedependent membrane hyperpolarization and cell death in leukemia cells. Blood. 2010;116(18):3593-603.

12. Melotti A, et al. The river blindness drug Ivermectin and related macrocyclic lactones inhibit WNT-TCF pathway responses in human cancer. EMBO Mol Med. 2014;6(10):1263-78.

13. Dou Q, et al. Ivermectin Induces Cytostatic Autophagy by Blocking the PAK1/Akt Axis in Breast Cancer. Cancer Res. 2016;76(15):4457-69.

14. Hashimoto $\mathrm{H}$, et al. Ivermectin inactivates the kinase PAK1 and blocks the PAK1-dependent growth of human ovarian cancer and NF2 tumor cell lines. Drug Discov Ther. 2009;3(6):243-6.

15. Evan Gl, Vousden KH. Proliferation, cell cycle and apoptosis in cancer. Nature. 2001;411(6835):342-8

16. Vyas $\mathrm{S}$, Zaganjor E, Haigis MC. Mitochondria and Cancer. Cell. 2016;166(3):555-66.

17. Kang $\mathrm{XH}$, et al. Degradation of Mcl-1 through GSK-3 beta Activation Regulates Apoptosis Induced by Bufalin in Non-Small Cell Lung Cancer H1975 Cells. Cell Physiol Biochem. 2017:41(5):2067-76.

18. Long $L$, et al. Silencing of GbANS reduces cotton resistance to Verticillium dahliae through decreased ROS scavenging during the pathogen invasion process. Plant Cell Tissue Organ Cult. 2018;135(2):213-21.

19. Bao D, et al. Protective Effect of Quercetin against Oxidative StressInduced Cytotoxicity in Rat Pheochromocytoma (PC-12) Cells. Molecules. 2017;22(7):1122

20. Cheng XS, et al. Neuronal Apoptosis in the Developing Cerebellum. Anat Histol Embryol. 2011;40(1):21-7.

21. Fulda S. Targeting apoptosis for anticancer therapy. Semin Cancer Biol. 2015;31:84-8.

22. Calviello G, et al. DNA damage and apoptosis induction by the pesticide Mancozeb in rat cells: involvement of the oxidative mechanism. Toxicol Appl Pharmacol. 2006;211(2):87-96.

23. Song $\mathrm{D}$, et al. Ivermectin inhibits the growth of glioma cells by inducing cell cycle arrest and apoptosis in vitro and in vivo. J Cell Biochem. 2019;120(1):622-33.

24. Real $\mathrm{R}$, et al. Involvement of breast cancer resistance protein (BCRP/ $A B C G 2)$ in the secretion of danofloxacin into milk: interaction with ivermectin. J Vet Pharmacol Ther. 2011;34(4):313-21.

25. Karin M. Nuclear factor-kappaB in cancer development and progression. Nature. 2006:441(7092):431-6.

26. Gong H, et al. Downregulation of miR-138 sustains NF-kappaB activation and promotes lipid raft formation in esophageal squamous cell carcinoma. Clin Cancer Res. 2013;19(5):1083-93.

27. Kausar T, et al. Clinical significance of GPR56, transglutaminase 2, and NF-kappaB in esophageal squamous cell carcinoma. Cancer Investig. 2011;29(1):42-8.

28. Chen Y, et al. Epigenetically upregulated oncoprotein PLCE1 drives esophageal carcinoma angiogenesis and proliferation via activating the PI-PLCepsilon-NF-kappaB signaling pathway and VEGF-C/ BCl-2 expression. Mol Cancer. 2019;18(1):1.

29. Deng H, et al. Combining alpha-Hederin with cisplatin increases the apoptosis of gastric cancer in vivo and in vitro via mitochondrial related apoptosis pathway. Biomed Pharmacother. 2019;120:109477.

30. Cui L, et al. Apoptosis induction by alantolactone in breast cancer MDAMB-231 cells through reactive oxygen species-mediated mitochondriondependent pathway. Arch Pharm Res. 2018;41(3):299-313.

31. Chen $L$, et al. Ivermectin suppresses tumour growth and metastasis through degradation of PAK1 in oesophageal squamous cell carcinoma. J Cell Mol Med. 2020;24(9):5387-401.

\section{Publisher's Note}

Springer Nature remains neutral with regard to jurisdictional claims in published maps and institutional affiliations.

Ready to submit your research? Choose BMC and benefit from:

- fast, convenient online submission

- thorough peer review by experienced researchers in your field

- rapid publication on acceptance

- support for research data, including large and complex data types

- gold Open Access which fosters wider collaboration and increased citations

- maximum visibility for your research: over $100 \mathrm{M}$ website views per year

At BMC, research is always in progress.

Learn more biomedcentral.com/submissions 